\title{
Development and Analysis of the Numerical Criterion for the Degree of Observability of State Variables in Nonlinear Systems
}

Proletarsky, A.V., Konstantin, A., Neusypin, K.S., Selezneva, M.S., and Grout, V.

This is a paper presented at the 7th IEEE Int. Conference on Internet Technologies and Applications ITA-17, Wrexham, UK, 12-15 September 2017.

Copyright of the author(s). Reproduced here with their permission and the permission of the conference organisers.

\section{Recommended citation:}

Proletarsky, A.V., Konstantin, A., Neusypin, K.S., Selezneva, M.S., and Grout, V. (2017) 'Development and Analysis of the Numerical Criterion for the Degree of Observability of State Variables in Nonlinear Systems'. In: Proc. 7th IEEE Int. Conference on Internet Technologies and Applications ITA-17, Wrexham, UK, 12-15 September 2017, pp. 150-154. doi: 10.1109/ITECHA.2017.8101927. 


\section{Development and Analysis of the Numerical Criterion for the Degree of Observability of State Variables in Nonlinear Systems}

\author{
Andrey V. Proletarsky, Konstantin A. Neusypin, \\ Kai Shen, Maria S. Selezneva \\ Faculty of Computer Science and Control Systems \\ Bauman Moscow State Technical University \\ Moscow, Russian Federation \\ shenkaichn@mail.ru
}

\author{
Vic Grout \\ Glyndwr University \\ Wrexham, UK \\ v.grout@glyndwr.ac.uk
}

\begin{abstract}
This paper is concerned with the problem of development and analysis of numerical criteria for the degree of observability in nonlinear systems. The disadvantages of existing criteria of observability and controllability were introduced. A numerical criterion for the degree of observability of each state variable was developed in nonlinear systems by utilizing the representation of nonlinear models in the State Dependent Coefficient form. The application of the novel criterion was demonstrated for the analysis of the degree of observability of inertial navigation system errors by the simulation with experimental data.
\end{abstract}

Keywords- nonlinear system, state dependent coefficient, the degree of observability, observation quality, navigation system

\section{INTRODUCTION}

In control systems theory, the problem of determining observability during the study of the properties of dynamic objects is essential. Generally, we only know the criteria for observability $[1,2]$ that allows determining which of the component of one state vector is observed better, compared with other components of the state vector. Therefore, these approaches give only a relative assessment of specific components of the state vector in the system under consideration and are inconvenient to apply when comparing the observation quality in the general case.

Usually, in practical applications, it is necessary to know the likelihood of effective observation of each specific component of the state vector. This is the reason for the introduction of the concept of the degree of observability $[3,4]$ for each state variable. Ablin proposed a criterion for the degree of observability based on the analysis of relationships between the estimation error values and observation (measurement) error values [3]. Ham and Brown then proved that eigenvalues and eigenvectors of the covariance matrix of estimation errors could provide useful information about the observability of the system [3]. From the point of view of the accuracy of estimation, the degree of observability can be determined by the ratio of the variance of a specific component of the state vector and the variance of a directly measured state variable [4]. They are criteria for the degree of observability of state variables considered in the linear formulation of the problem. To determine the degree of observability of specific state variables in nonlinear systems, it is necessary to develop a new numerical criterion.

In this paper, we first derive a novel numerical criterion for the degree of observability of specific state variables in nonlinear systems. Then, the possibility of applying the criterion for the degree of observability is demonstrated by determining the quantitative characteristics of observability when estimating errors of an inertial navigation system (INS) with a real system Ts- 060 .

\section{OBSERVABILITY CRITERIA IN NONLINEAR SYSTEMS}

Let the error model of the inertial navigation system be described by the differential equations

$$
\begin{aligned}
& \frac{d}{d t} x(t)=f(t, x)+g(t, x) w(t), x\left(t_{0}\right)=x_{0}, \\
& y(t)=h(t, x)+v(t) . \\
& f, g: T \times \Omega_{x} \rightarrow R^{n}, h: T \times \Omega_{x} \rightarrow R^{m}, \\
& (t, x) \rightarrow f(t, x), g(t, x), h(t, x) .
\end{aligned}
$$

where $T$ is the interval $\left[t_{0}, t_{1}\right] ; x(t) \in \Omega_{x}$, where $\Omega_{x}$ is a space (open related set) $R^{n} ; x \in R^{n}$ is the state of the system; $x_{0} \in \Omega_{x} ; w \in R^{n}$ is the input noise; $y \in R^{m}, m \leq n$ is the system measurement; $v \in R^{m}$ is the measurement noise; matrices $f(t, x), g(t, x), h(t, x)$ are valid and continuous.

Let us consider the system (1) in an equivalent form: the model has the structure of linear differential equations with parameters that depend on the state (State Dependent Coefficient, SDC) [5]. The representation of equations in (1) transformed by utilizing the SDC method has the form

$$
\begin{aligned}
& \frac{d}{d t} x(t)=A(t, x) x(t)+G(t, x) w(t), x\left(t_{0}\right)=x_{0}, \\
& y(t)=H(t, x) x(t)+v(t) .
\end{aligned}
$$


where $A(\cdot), G(\cdot), H(\cdot)$ are matrices of real variables.

The SDC representation of the nonlinear system (1) is observed in $T \times \Omega_{x}$ if the pair $\langle A(t, x) H(t, x)\rangle$ is linearly observable [6] for $(t, x) \in T \times \Omega_{x}$, i.e.

$$
\operatorname{rank}[O(t, x)]=\operatorname{rank}\left[\begin{array}{c}
H(t, x) \\
H(t, x) A(t, x) \\
\cdots \\
H(t, x) A(t, x)^{n-1}
\end{array}\right]=n .
$$

This means that there is a positive definite matrix $\Gamma(t, x)$ (observability Gramian) for any $(t, x) \in T \times \Omega_{x}$, which is a solution of the Lyapunov's equation

$$
A^{T}(t, x) \Gamma(t, x)+\Gamma(t, x) A(t, x)+H^{T}(t, x) H(t, x)=0 .
$$

This criterion is similar to Kalman's criterion of observability [6] for linear systems.

It should be noted that the study of the observability in the systems of the form (2), i.e. systems with parameters depending on the state, does not currently have common constructive solutions. The study of such systems is generally limited to checking compliance with the conditions of observability "on the point", i.e. in the neighborhood of the studied state for the linearized system.

In practice, for information processing, the system is often expressed in a discrete form. In a discrete form, the SDC representation of the nonlinear system (2) has the form

$$
\begin{gathered}
x_{k+1}=\Phi\left(t_{k}, x_{k}\right) x_{k}+G\left(t_{k}, x_{k}\right) w_{k}, \\
y_{k+1}=H\left(t_{k+1}, x_{k+1}\right) x_{k}+v_{k+1} .
\end{gathered}
$$

It is assumed that $w_{k}$ and $v_{k+1}$ are Gaussian white uncorrelated noise, and for any $j$ and $k, v_{j}$ and $w_{k}$ are not correlated (i.e. $E\left[v_{j} w_{k}^{T}\right]=0$ ).

Considering the system (5), we can easily get

$$
\begin{aligned}
y_{k}= & H\left(t_{k}, x_{k}\right) x_{k}+v_{k}, \\
y_{k+1}= & H\left(t_{k+1}, x_{k+1}\right) \Phi\left(t_{k}, x_{k}\right) x_{k}+H\left(t_{k+1}, x_{k+1}\right) w_{k}+v_{k+1}, \\
& \cdots \quad \cdots \quad \quad \cdots \\
y_{k+n-1}= & H\left(t_{k+n-1}, x_{k+n-1}\right) \Phi\left(t_{k+n-2}, x_{k+n-2}\right) \cdots \Phi\left(t_{k}, x_{k}\right) x_{k}+ \\
& +H\left(t_{k+n-1}, x_{k+n-1}\right) \Phi\left(t_{k+n-2}, x_{k+n-2}\right) \cdots \Phi\left(t_{k+1}, x_{k+1}\right) w_{k}+ \\
& +\cdots+H\left(t_{k+n-1}, x_{k+n-1}\right) w_{k+n-2}+v_{k+n-1} .
\end{aligned}
$$

or in a matrix form as

$$
y_{k}^{*}=O_{k}^{*} x_{k}+v_{k}^{*}
$$

where $y_{k}^{*}=\left[\begin{array}{c}y_{k} \\ y_{k+1} \\ \vdots \\ y_{k+n-1}\end{array}\right]$

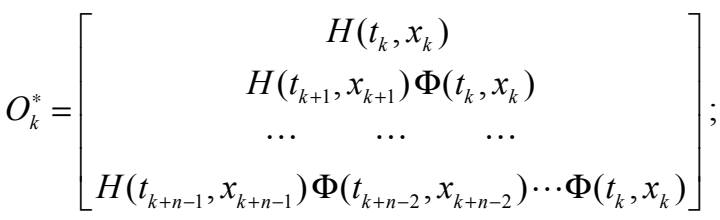

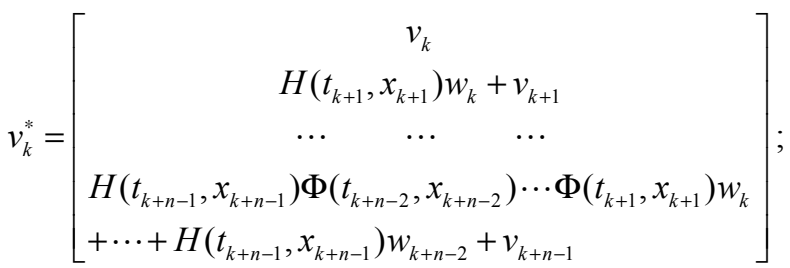

vectors $y_{k}^{*}, v_{k}^{*}$ and matrix $O_{k}^{*}$ include parameters that depend on the state.

The matrix $O_{k}^{*}$ in the formula (7) for nonlinear systems is the matrix of observability. In accordance with the criterion (3), the system (5) is observable if $\operatorname{rank}\left[O_{k}^{*}\right]=n$, i.e. all columns in the matrix $O_{k}^{*}$ are independent.

\section{NUMERICAL CRITERION FOR OBSERVABILITY IN NONLINEAR SYSTEMS}

The existing criteria for the degree of observability [3, 4] were proposed for system analysis in linear time invariant and time-varying systems. However, there are situations when the objects of analysis have a clear nonlinear form. In this case, to determine the degree of observability of state variables in nonlinear systems, it is necessary to modify the existing criteria.

Taking the equation (7) into account, we obtain

$$
O_{k}^{* T} y_{k}^{*}=O_{k}^{* T} O_{k}^{*} x_{k}+O_{k}^{* T} v_{k}^{*} \text {. }
$$

Thus, the equation (8) would be

$$
O_{k}^{* T} O_{k}^{*} x_{k}=O_{k}^{* T} y_{k}^{*}-O_{k}^{* T} v_{k}^{*}
$$

When the SDC representation of the nonlinear system (2) is observable, the rank of the observability matrix $O_{k}^{*}$ equals to the order of the system $n$, so the rank of matrix $O_{k}^{* T} O_{k}^{*}$ is also the order of the system $n$. Hence, the matrix $O_{k}^{* T} O_{k}^{*}$ is invertible. Therefore, we can obtain

$$
x_{k}=\left[O_{k}^{* T} O_{k}^{*}\right]^{-1} O_{k}^{* T} y_{k}^{*}-\left[O_{k}^{* T} O_{k}^{*}\right]^{-1} O_{k}^{* T} v_{k}^{*} .
$$


Now, we introduce a designation

$$
\zeta_{k}=O_{k}^{+} y_{k}^{*}
$$

where $O_{k}^{+}=\left[O_{k}^{* T} O_{k}^{*}\right]^{-1} O_{k}^{* T}$ is the pseudoinverse matrix of $O_{k}^{*}$.

It should be noted that the matrix $O_{k}^{+}$is also a matrix with parameters depending on the state.

In practical application, it is usually necessary to determine the degree of observability of state variables during the operation of a dynamic object. For simplicity, we express the equation (11) in a scalar form as

$$
\zeta_{k}^{i}=\alpha_{1}^{i} y_{k}+\alpha_{2}^{i} y_{k+1}+\cdots+\alpha_{n}^{i} y_{k+n-1}
$$

where $\zeta_{k}^{i}$ is the $i$-th element of the vector $\zeta_{k}, \alpha_{j}^{i}(j=1, \cdots, n)$ is from the $i$-th row of the matrix $O_{k}^{+}$.

In accordance with the equation (12), the equation of measurement is formed as

$$
v_{k}^{i}=\alpha_{1}^{i} v_{k}+\alpha_{2}^{i} v_{k+1}+\cdots+\alpha_{n}^{i} v_{k+n-1}
$$

Therefore, the variance of the measurement noise, given the $i$-th component, has the form as

$$
\Upsilon_{k}^{i}=E\left[\left(v_{k}^{i}\right)^{2}\right]=\left[\left(\alpha_{1}^{i}\right)^{2}+\left(\alpha_{2}^{i}\right)^{2}+\cdots+\left(\alpha_{n}^{i}\right)^{2}\right] r_{k}
$$

where $r_{k}=E\left[v_{k}^{2}\right]$ is the variance of the original measurement noise $v_{k}$.

In order to develop a criterion for the degree of observability of state variables in nonlinear systems, we use the structure of the criterion [4] for linear systems.

The numerical criterion for the degree of observability in nonlinear systems has the following form

$$
\begin{aligned}
\Lambda_{k}^{i}= & \frac{M\left[\left(x_{k}^{i}\right)^{2}\right] r_{k}}{M\left[\left(\zeta_{k}^{i}\right)^{2}\right] \Upsilon_{k}^{i}} \\
= & \frac{M\left[\left(x_{k}^{i}\right)^{2}\right]}{M\left[\left(\zeta_{k}^{i}\right)^{2}\right] \sum_{j=1}^{n}\left(\alpha_{j}^{i}\right)^{2}},
\end{aligned}
$$

where $E\left[\left(x_{k}^{i}\right)^{2}\right]$ is the variance of the $i$-th component of the state vector; $E\left[\left(\zeta_{k}^{i}\right)^{2}\right]$ is the variance of the measurement.
Here, it should be noted that during the study of such nonlinear systems it is necessary to calculate the degree of observability "on the point" [6]. That is to say, it is essential to consider the influence of parameters that depend on the state in vectors $y_{k}^{*}, v_{k}^{*}$ and the matrix $O_{k}^{+}$.

\section{SimUlation AND ANALYsis}

Error equations of an inertial navigation system Ts-060 are the error equations of orientation and equations of the errors of horizontal accelerometers.

These equations have the form as [7]

$$
\begin{aligned}
\delta \dot{V} & =-g \psi+B \\
\dot{\psi} & =\frac{\delta V}{R}-\frac{\delta V}{R} \psi+\varepsilon \\
\dot{\varepsilon} & =-\mu \varepsilon+\eta
\end{aligned}
$$

where $\delta V$ is the error in determining the velocity; $\psi$ is the deflection angle of the gyro-stabilized platform (GSP); $\varepsilon$ is the drift rate of the GSP; $B, \eta$ are Markov random processes; $R$ is the radius of the Earth; $g$ is the acceleration due to gravity; $\mu$ is the average frequency of random changes of drift rate.

Equations (16) in a matrix form are as follows

$$
\dot{x}(t)=f(t, x(t))+w(t)
$$

where $x(t)=\left[\begin{array}{l}x_{1} \\ x_{2} \\ x_{3}\end{array}\right]=\left[\begin{array}{c}\delta V \\ \psi \\ \varepsilon\end{array}\right] ; f(t, x)=\left[\begin{array}{c}-g x_{2} \\ \frac{x_{1}}{R}+\frac{x_{1} x_{2}}{R}+x_{3} \\ -\mu x_{3}\end{array}\right]$; $w(t)=\left[\begin{array}{c}B \\ 0 \\ \eta\end{array}\right]$

In accordance with the equation (2), we obtain the SDC representation of the equation (17) as

$$
\dot{x}(t)=A(t, x) x(t)+w(t),
$$

where $A(t, x) x(t)=\left[\begin{array}{ccc}0 & -g & 0 \\ \frac{1}{R} & \frac{x_{1}}{R} & 1 \\ 0 & 0 & -\mu\end{array}\right]\left[\begin{array}{l}x_{1} \\ x_{2} \\ x_{3}\end{array}\right]$

In a discrete form, the SDC representation of the nonlinear system (18) has a form as 


$$
x_{k+1}=\Phi_{k+1, k} x_{k}+w_{k},
$$

where $x_{k}=\left[\begin{array}{c}\delta V_{k} \\ \psi_{k} \\ \varepsilon_{k}\end{array}\right] ; w_{k}=\left[\begin{array}{c}B_{k} \\ 0 \\ \eta_{k}\end{array}\right]$;

$\Phi_{k+1, k}=\left[\begin{array}{ccc}1 & -T g_{k} & 0 \\ \frac{T}{R_{k}} & 1+\frac{T \delta V_{k}}{R_{k}} & T \\ 0 & 0 & 1-T \mu\end{array}\right]$,

$T$ is the sampling period.

During the operation of aircrafts, the INS errors can reach significant values. Generally, the compensation of INS errors is carried out algorithmically with Global Navigation Satellite Systems (GNSS). With this condition, the measurement equation has a form as

$$
z_{k+1}=H_{k+1} x_{k+1}+v_{k+1}
$$

where $z_{k+1}=V_{k+1}^{I N S}-V_{k+1}^{G N S S}=\delta V_{k+1}+v_{k+1}^{G N S S}$, i.e. $v_{k+1}=v_{k+1}^{G N S S}$ is a Markov random process; $H_{k}=\left[\begin{array}{ccc}1 & 0 & 0\end{array}\right]$.

In accordance with the equation (7), we obtain the matrix of observability as

$$
O_{k}^{*}=\left[\begin{array}{ccc}
1 & 0 & 0 \\
1 & -T g_{k} & 0 \\
1-\frac{T^{2} g_{k+1}}{R_{k}} & -T g_{k}-T g_{k+1}-\frac{T^{2} g_{k+1} x_{1, k}}{R_{k}} & -T^{2} g_{k+1}
\end{array}\right]
$$

For the simulation, we used the data from a semi-natural experiment with the real INS Ts-060 mounted on a fixed base. The INS's output signals are the errors in determination of velocity and are used as the measurement for the estimation algorithm. In this case, the laboratory experiment was conducted using a nonlinear Kalman filter. The simulation results are presented in figures 1 and 2.

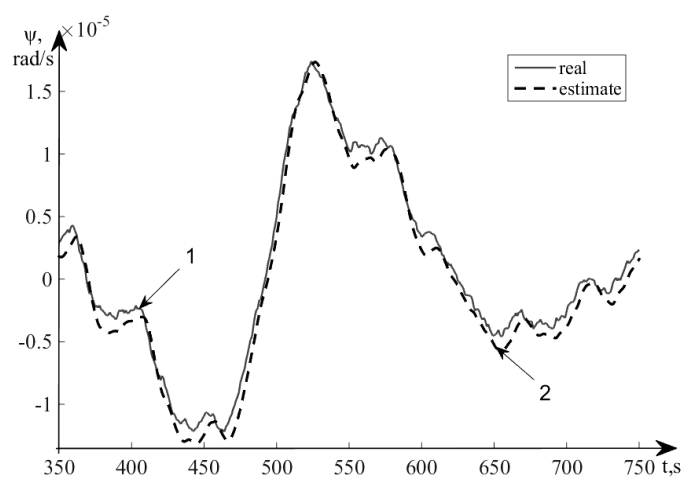

Fig. 1. The simulation result of estimation of the deflection angle

In Figure 1, solid-line 1 - real values during the laboratory experiment, broken-line 2 - estimates of the deflection angle with classical nonlinear Kalman filtering.

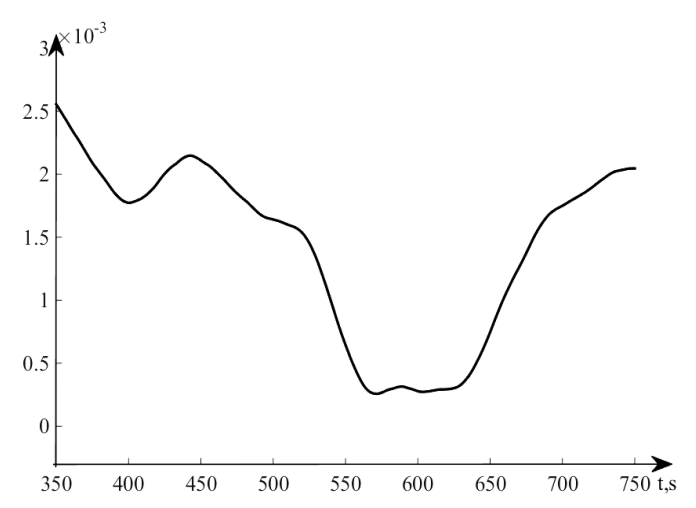

Fig. 2. The degree of observability of the deflection angle

Numerical values of the degree of observability change over time, since they depend on the estimates of state variables and model parameters. The numerical values are consistent with the data based on the analysis of the degree of observability of the INS errors. What's more, the proposed criterion can be easily employed in practice with less computer memory and more clear physical meaning.

\section{CONCLUSIONS}

To determine the quantitative characteristics of observability of state variables in nonlinear systems, an original numerical criterion for the degree of observability is proposed. The basis of the developed criterion is the representation of the nonlinear model in the State Dependent Coefficient form and the structure of the known criterion for the degree of observability of state variables in linear systems. The application of the novel criterion is demonstrated for the analysis of the degree of observability of inertial navigation system errors via the simulation with experimental data

\section{ACKNOWLEDGMENT}

This research was supported by the Russian Foundation for Basic Research (Project No. 16-8-00522) and the Programme of Introducing Talents of Discipline to Universities in P.R. China ("111 program" No. B 16025).

\section{REFERENCES}

[1] R. Hermann, A.J. Krener, "Nonlinear controllability and observability", IEEE Transactions on Automatic Control, Vol. AC-22, No 5, pp. 728740, 1977.

[2] D. Goshen-Meskin, I. Bar-Itzhack, "Observability analysis of piece-wise constant systems. I. Theory", IEEE Transactions on Aerospace and Electronic Systems, Vol. 28, Iss. 4, pp. 1056-1067, 1992.

[3] F.M. Ham, R.G. Brown, "Observability, eigenvalues, and Kalman filtering", IEEE Transactions on Aerospace and Electronic Systems, Vol. AES-19, Iss. 2, pp. 269-273, 1983. 
[4] Kai Shen, K.A. Neusypin, A.V. Proletarsky, "On state estimation of dynamic systems by applying scalar estimation algorithms", Proceedings of 2014 IEEE Chinese Guidance, Navigation and Control Conference, Yantai, China, pp. 124-129, August, 2014.

[5] R.C. James, N.D. Christopher, P.M. Curtis, "Nonlinear regulation and nonlinear $\mathrm{H} \infty$ control via the state-dependent Riccati equation technique: Part 1, Theory", Proceedings of the First International Conference on
Nonlinear Problems in Aviation and Aerospace, Daytona Beach, FL USA, pp. 117-141, 1996.

[6] V.N. Afanas'ev, "Control of nonlinear plants with state-dependent coefficients", Automation and Remote Control, Vol. 72, No.4, pp. 713 726, 2011.

[7] Kai Shen, A.V. Proletarsky, K.A. Neusypin, "Quantitative analysis of observability in linear time-varying systems", Chinese Control Conference, Chengdu, China, pp. 44-49, July 2016. 